\title{
Role of altered gut microbiota in the development of obesity, insulin resistance and type 2 diabetes mellitus
}

\author{
Shrestha $U K^{1^{*}}$
}

${ }^{1}$ Department of Medicine, Manipal College of Medical Sciences, Pokhara, Nepal

*Corresponding Author:

Dr. Umid Kumar Shrestha, MD, PhD Department of Medicine

Manipal College of Medical Sciences

\& Manipal Teaching Hospital

Pokhara, Nepal

Email: umidshrestha@gmail.com

\section{Citation}

Shrestha UK. Role of altered gut microbiota in the development of obesity, insulin resistance and type 2 diabetes mellitus. Nepal journal of Medical Sciences. 2013;2(1):1-2.
The prevalence of obesity and obesity-associated disorders, such as insulin resistance, type 2 diabetes mellitus, cardiovascular disease and others have increased significantly worldwide over the last decade. The obesity is caused by a complex process derived from the interaction of largely unknown multiple genetic and environmental factors. In such circumstances, the gut microbiota or microflora has been increasingly recognized as an important factor in development of obesity by interplay with the genes, environment, and immune system. The human gut microbiota, which refers to the microorganisms residing in the intestine and is integral in multiple physiological processes of the host, is estimated to consist of at least $10^{14}$ bacteria and archaea, composed of approximately 1,100 prevalent species, with approximately 160 such species per individual. ${ }^{1}$ Gut microbiota could now be considered as a "microbial organ" localized within the host. The genome size of this microbial organ, collectively named microbiome, exceeds the size of the human nuclear genome by two orders of magnitude and provides important biological and metabolic functions. Mammalian gut microbiota belongs predominantly to four bacterial phyla: the Gram-negative Bacteroidetes and Proteobacteria and the Grampositive Actinobacteria and Firmicutes.

Recently, the study demonstrated the role of gut microbiota in insulin resistance in Toll-like receptor (TLR)-2 knockout mice. ${ }^{2}$ The correlation of altered microbiota composition with the obesity, insulin resistance, and diabetes has been demonstrated in the host through several mechanisms: increased energy harvest from the diet, altered fatty acid metabolism and composition in adipose tissue and liver, modulation of gut peptide YY and glucagon-like peptide (GLP)-1 secretion, activation of the lipopolysaccharide TLR-4 axis, and modulation of intestinal barrier integrity by GLP-2. ${ }^{3}$ Several factors modify the composition of gut microflora, such as: mode of delivery, type of infant feeding, hospitalization, and prematurity, the antibiotic use and dietary nutrient composition, and the Western diet; the latter may promote an obesogenic gut microbiota by causing low grade chronic inflammation. ${ }^{3}$ Several studies have showed the benefit of prebiotics and probiotics on insulin sensitivity, inflammatory markers, and glucose tolerance. ${ }^{4-6}$

Recently, one study was done to study the effects of transferring intestinal microbiota from healthy, lean men (body mass index $<23 \mathrm{~kg} / \mathrm{m}^{2}$ ) to men with metabolic syndrome (obesity, high blood 
pressure, and increased blood levels of glucose or insulin resistance). ${ }^{7}$ In recipients of microbiota from lean donors, bacterial groups that increased significantly included those related to the butyrate-producer Roseburia intestinalis, which increased 2.5-fold, the oxalate-converting Oxalobacter formigenes, and other Firmicutes. Also, levels of fecal short-chain fatty acids decreased after infusion of microbiota from lean donors. Insulin sensitivity could be increased by butyrate production and greater intestinal microbiota diversity could be associated with a less insulin resistance. These findings could pave the way for a new therapeutic approach for diabetes. ${ }^{7}$

Studies have shown that the infusion of donor feces was significantly more effective for the treatment of recurrent $C$. difficile infection than the use of vancomycin. ${ }^{8}$ Hence, it can be speculated that the fecal transplants could also become another interesting option for the therapy of obesity and metabolic syndrome.

More research is needed to understand the hormonal, immunomodulatory, and metabolic mechanisms underlying microbe-microbe and microbiota-host interactions and the specific genes that determine the health benefit derived from probiotics. The relative impact of pre/probiotics on the treatment of obesity may be further explored by the future trials. However, it is still convincing that a friendly gut microbiota- achieved by a healthy lifestyle, which includes breast lactation, appropriate antibiotic use, and the avoidance of excessive dietary fat intake- may positively affect the prevention and treatment of obesity, insulin resistance and type 2 diabetes mellitus.

Keywords: Gut microbiota; obesity; insulin resistance

References:

1. Qin J, Li R, Raes J, et al. A human gut microbial gene catalogue established by metagenomic sequencing. Nature 2010;464:59-65.

2. Caricilli AM, Picardi PK, de Abreu LL, et al. Gut microbiota is a key modulator of insulin resistance in TLR 2 knockout mice. PLoS Biol 2011;9:e1001212.

3. Musso G, Gambino R, Cassader M. Obesity, diabetes, and gut microbiota: the hygiene hypothesis expanded? Diabetes Care 2010;33:2277-84.

4. Laitinen K, Poussa T, Isolauri E. Probiotics and dietary counselling contribute to glucose regulation during and after pregnancy: a randomised controlled trial. Br J Nutr 2009;101:1679-87.

5. Luoto R, Laitinen $\mathrm{K}$, Nermes $\mathrm{M}$, et al. Impact of maternal probioticsupplemented dietary counselling on pregnancy outcome and prenatal and postnatal growth: a double-blind, placebo-controlled study. Br J Nutr 2010;103:1792-9.

6. Crawford PA, Crowley JR, Sambandam N, et al. Regulation of myocardial ketone body metabolism by the gut microbiota during nutrient deprivation. Proc Natl Acad Sci USA 2009;27:11276-81.

7. Vrieze A, Van Nood E, Holleman F, et al. Transfer of intestinal microbiota from lean donors increases insulin sensitivity in individuals with metabolic syndrome. Gastroenterology 2012;143:913-6.e7.

8. van Nood E, Vrieze A, Nieuwdorp M, et al. Duodenal Infusion of Donor Feces for Recurrent Clostridium difficile. N Engl J Med 2013 Jan 16. [Epub ahead of print] 